\title{
A quantified description of the coupling between the reaching direction and elbow axis direction
}

doi:10.1080/11762320701455583

\author{
M. M. Stanišić ${ }^{1}$, C. M. Goehler ${ }^{1}$ and M. Tomšič ${ }^{2}$ \\ ${ }^{I}$ Department of Aerospace and Mechanical Engineering, University of Notre Dame, Notre Dame, IN 46556 USA \\ ${ }^{2}$ Department for Automatics, Biocybernetics and Robotics, Jošef Stefan Institute, Jamova 39, 1000 Ljubljana, Slovenia
}

\begin{abstract}
The human body continues to be an inspiration for the work of a myriad of different fields, both scientific and mathematic. In particular, robotics draws upon the motions and relationships of different human systems in order to reproduce similar mobility while performing different tasks. The human shoulder-arm-elbow complex has been one of the most difficult to exactly replicate. This paper examines the relationship between the motion of the shoulder while positioning the center of the wrist during voluntary arm movements and the resulting orientation of the arm, in particular the direction of the axis passing through the elbow joint. Experimental data is presented that was used to quantify this coupling between the reaching direction of the arm and the elbow axis direction. The results from this paper are two surface-fit equations that can be used to determine the elbow axis direction when given the location of the wrist center. These results are useful when considering the design and control of shoulder-arm-elbow complex models.
\end{abstract}

Key words: Human joint motion couplings, humanoids, robotics, human motion simulation.

\section{INTRODUCTION}

A new challenge in robotics is the development of humanoids, that is, machines that move in a human-like manner. A difficulty with humanoid design is the kinematic interpretation of human joints and the development of mechanisms that can mimic human motion. One step in overcoming this boundary is to gain a better understanding of how different human musculo-ligamental-skeletal systems operate and interact. The focus of this paper is on the human shoulder-arm-elbow complex and the couplings that exist therein.

As is the case with other complexes in the human body, there exist couplings between the different joints within the shoulder complex. The most notable coupling in the shoulder complex exists between the shoulder girdle and the glenohumeral joint. The different rotations of the glenohumeral joint have been found to be coupled with transla-

Correspondence Author:

M. M. Stanišić

Department of Aerospace and Mechanical Engineering

University of Notre Dame, Notre Dame

IN, 46556 USA

Tel: 011-574-631-7897, Fax: 011-574-631-8341

Email: stanisic@nd.edu tions of the humeral head (Novotny et al. 1998). An interesting aspect of this coupling is that none of these rotations and translations are planar. In other words, attempting to move the humerus in a defined plane or about a defined axis results in rotations or translations of the bones about an axis or in a plane other than the one initially intended. The large ranges of motion of the glenohumeral joint along with the coupled, spatial motion hinder the development of kinematic testing models. As it stands, these coupled motions are documented as mean values of data recorded from cadaver specimens, i.e. a certain mean translation of the humeral head occurs with a corresponding mean rotation of the humerus. There has been no general relationship between the translations due to the shoulder complex and the rotations of the glenohumeral joint agreed upon to date. However, it has been suggested that the relationship between the pitch of the shoulder girdle from the transverse plane and the angle from the humerus to the transverse plane during abduction and adduction can be approximated as a 1:3 ratio (Oatis 2004; Zatsiorsky 1998). This relationship is more commonly known as the shoulder rhythm.

Another less noted coupling involving the shoulder exists between the shoulder complex and the elbow joint. This coupling can be identified as the relationship between shoulder motions and the orientation of the primary axis of 
the elbow. It is this coupling that will be studied in depth in this paper.

Bosga et al. (2003) stated that the coupling of the joints of the arm is partially effected by the stabilizing role of poly-articular muscles, inertia and movement frequency. Using circular drawing movements, it was shown that the coupling between the shoulder and elbow rotations decoupled at certain locations in the mid-sagittal plane. This was due to specific joint-surface characteristics at different locations in the sagittal plane. The coupling was not disturbed by inertial or frequency effects, rather these factors affected the elbow-wrist coupling. The shoulder-elbow relationship was only observed in the experiments with no attempt to model the coupling. Debicki and Gribble (2004) also noted the existence of a shoulder-elbow coupling while performing experiments on the adaptation of the nervous system to different loads applied to the arm.

De Sapio et al. (2006) utilized skeletal kinematics, muscle routing kinematics and muscle strength characteristics to determine a muscle effort criteria for predicting human arm motions. Their modeling of the shoulder rhythm used constraints between the scapula, clavicle and humerus that were determined from the shoulder rhythm regression analysis of de Groot and Brand (2001). The proposed criteria by De Sapio et al. was found to properly account for the cross-joint coupling associated with poly-articular muscle routing kinematics and showed good correlation between natural reaching postures and those predicted by their dynamic model.

This paper examines human arm motions while performing reaching tasks. The end result is a quantified coupling between two vectors (the reaching direction and elbow axis direction) which is based solely on experimental data and does not require a priori knowledge of the skeletal or muscular kinematics of the human shoulder-arm-elbow complex.

\section{DEFINITION OF COORDINATE SYSTEM AND ANATOMICAL PLANES}

Three-dimensional analysis of human body kinematics requires a coordinate system, typically composed of anatomically aligned axes as shown in Figure 1: medial/lateral (ML), anterior/posterior (AP) and superior/inferior (SI) (Oatis 2004). The following Cartesian coordinate system is used throughout this paper: the $\mathrm{X}$ axis is aligned with the ML axis with positive $X$ in the medial direction, the $Y$ axis is aligned with the $\mathrm{SI}$ axis with positive $\mathrm{Y}$ in the superior direction and the $Z$ axis is aligned with the AP axis with positive $\mathrm{Z}$ in the anterior direction. This coordinate system will be referred to as the anatomical body frame.

In the human body, there are three perpendicular anatomical planes referred to as the cardinal planes. The sagittal plane is the $\mathrm{Y}-\mathrm{Z}$ plane, the frontal plane is the $\mathrm{X}-$ $\mathrm{Y}$ plane and the transverse plane is the $\mathrm{X}-\mathrm{Z}$ plane. These planes are also displayed in Figure 1 (Oatis 2004).

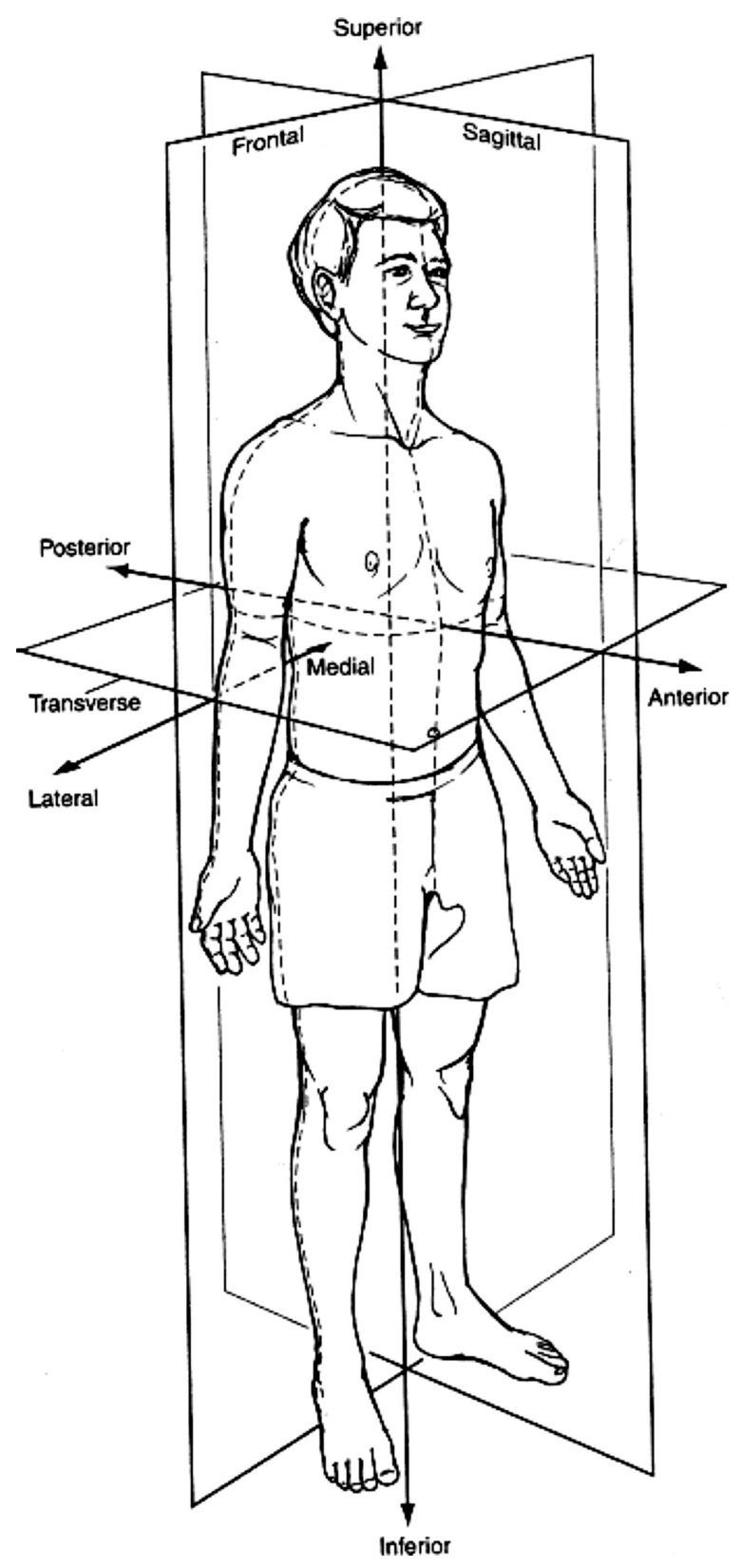

Figure 1 Definition of cardinal planes (Oatis 2004).

\section{VOLUNTARY ARM MOVEMENTS}

Due to the excess of degrees of freedom in the human shoulder-arm complex, a position of the hand can be reached by many joint configurations (Gielen et al. 1997). Based on observations that movements with the same beginning and end points were made in a consistent way with the same joint configurations as a function of time, Gielen et al. (1997) suggested that there is a reduction of the number of degrees of freedom, or coupling, during reaching motions of the human arm. These similar joint configurations represent voluntary arm movements (Flash and Hogan 1985).

One of the central issues for understanding how the human body works is how the excessive degrees of freedom of 
this neuromotor system are controlled during these common, everyday movements (Zaal et al. 1999). Such movements require coordination of numerous skeletal joints and muscles leading to couplings within different systems of the body. Zaal et al. (1999) suggested that the shoulder-elbow coupling exists in the sagittal plane due to a proportionality between muscle torques at the shoulder and muscle torques at the elbow.

Cruse and Brüwer (1987) studied a redundant manipulator in the transverse plane free to move at the shoulder, elbow and wrist joints. Cost functions were assigned to each joint associated with "comfortable" joint angles, which was at a minimum at about the middle of the angle range of the joint. It was concluded that the control system of the movement can be interpreted as a compromise between four requirements, (a) an equal contribution of all joints to the movement, (b) minimization of the static costs by means of the cost functions, (c) minimizing the inertial forces acting at the tip of the end effector by following a straight workspace path, and (d) simplifying the pattern of muscle activity. The resulting relationship between the angular displacements in the shoulder and elbow was mostly linear but occasionally of a polynomial nature. This suggests that the coupling between the two rotations in the transverse plane changes depending on not only the travel distance, but where the beginning and ending points are located relative to the torso.

Flash and Hogan (1985) studied the coordination of voluntary human arm movements. Trajectories yielding the best performance were determined by minimizing the square of the magnitude of jerk of the hand integrated over the entire movement. This assumes that a major goal of coordination is the production of the smoothest possible movement of the hand. The theoretical model was verified through experimental observations of human subjects performing unconstrained, or voluntary, movements in the transverse plane.

There have been several attempts to reproduce these voluntary arm movements using varying techniques. Potkonjak et al. (1998) analyzed the motion of a redundant anthropomorphic arm while writing. A model of their experimental setup can be seen in Figure 2. The modeling is based on distributed positioning, which is the separation of the prescribed movement in smooth global motion and fast local motion. The suggested approach allows for unique solutions of the inverse kinematics of redundant mechanisms such as the human arm or anthropomorphic robot arm. The experimental and theoretical results were quite similar. The workspace of the tested motion was two-dimensional and assumed a relationship between the shoulder and elbow allowing for the authors to reduce the degrees-of-freedom of the system.

Kieffer and Lenarcic (1994) showed how humans use singularity configurations of their limbs to gain mechanical advantage and investigated the possibility of obtaining similar benefits in robotic systems. It was shown that using a minimization of joint torques in redundant sys-

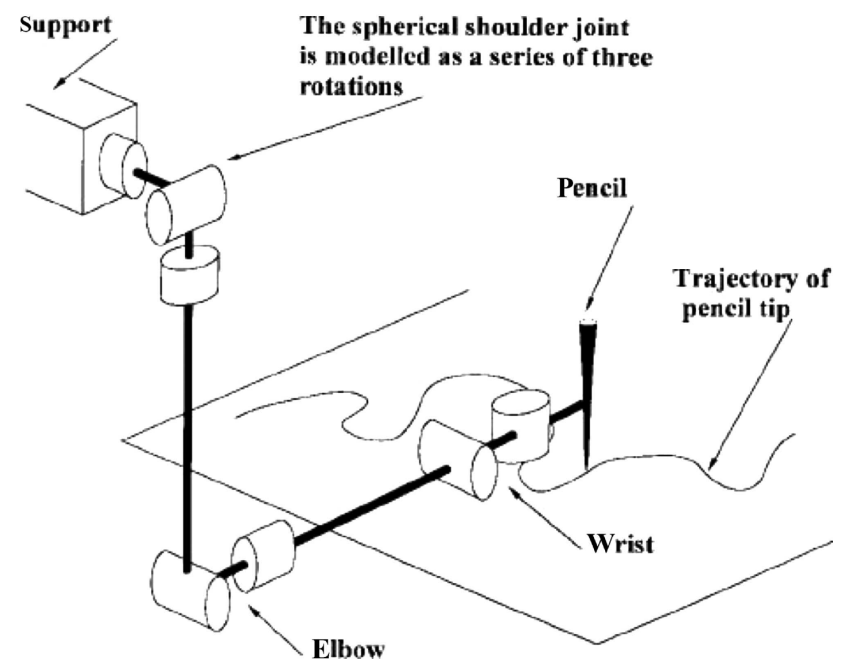

Figure $27-\mathrm{DOF}$ anthropomorphic arm in writing task (Potkonjak et al. 1998).

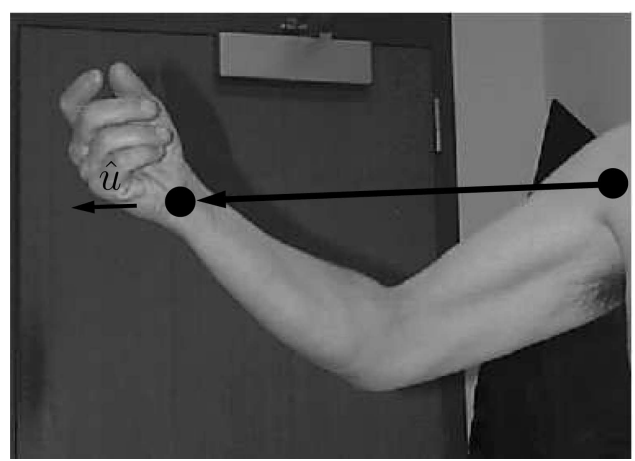

Figure 3 Definition of the reaching direction.

tems led to human-like behavior that favored singularities. This method was applied to planar systems, and stable implementation of such behaviors requires a strategy which gives the robot more autonomy with respect to timing task execution.

\section{SHOULDER-ELBOW MOTION COUPLING}

In this paper, a reaching direction is defined as the unit vector, $\hat{u}$, in the direction of a vector originating at the shoulder center and terminating at the wrist center as shown in Figure 3.

\section{Quantifying the shoulder-elbow coupling}

This paper proposes that for voluntary arm movements:

- There exists one orientation of the elbow axis for each wrist position.

- Every wrist position along one reaching direction has the same corresponding elbow axis direction. These two vectors are displayed in Figure 4.

Utilizing these assumptions simplifies the coupling description. Given the position of the wrist center, the reaching direction can be calculated. The direction of the elbow 


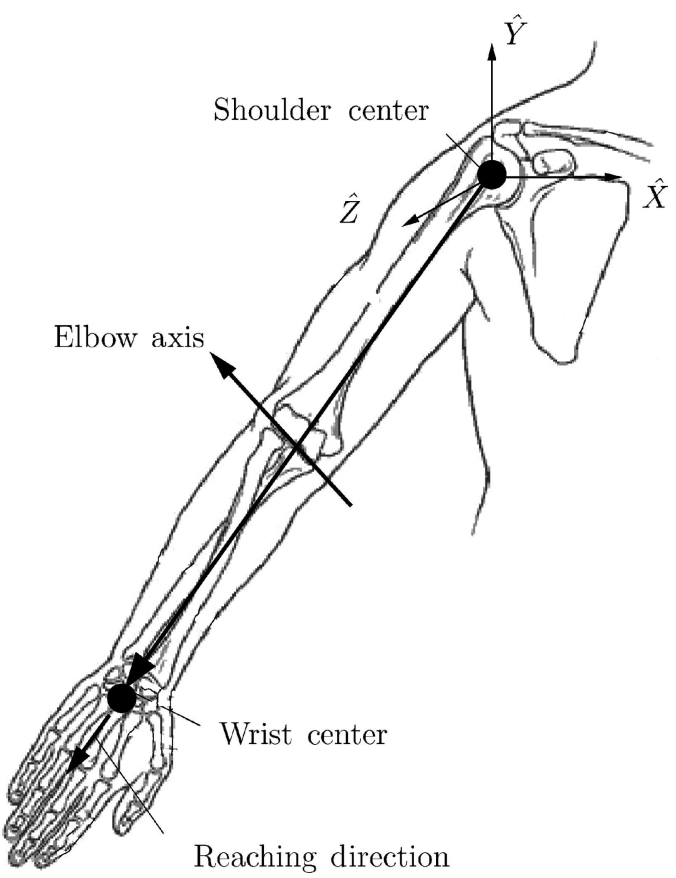

Figure 4 Vectors of interest for the shoulder-elbow coupling [original arm figure (McCabe and Goldman 2000)].

axis is determined by a function based on the reaching direction. This function was determined by fitting a surface to data points using regression analysis.

In order to both prove the hypotheses stated above and gather enough data for a effective three-dimensional regression analysis fit, motion studies were performed at the Jošef Stefan Institute utilizing a BTS SMART motion capture system.

\section{Subjects}

Eleven subjects were recorded during the motion studies: nine males aging 29, 30 (2), 31, 33, 39, 44 and 45 (2); and two females aging 28 and 33 . None of the subjects had any history of neuromuscular disorders or motor impairments.

\section{Experimental conditions}

These subjects were fitted with six reflective markers in order to record their arm movements as seen in Figure 5: two markers on the sternum, one approximately at the shoulder center, two lying on the axis of rotation of the elbow and one approximately at the wrist center. In order to be able to relate the marker coordinates to the anatomical body frame defined in the second section, a set of markers forming a coordinate system were initially recorded for each subject with the origin located approximately at the shoulder center.

Each subject performed a set amount of trajectories and static poses. Right hand motion was recorded. The trajectories and static poses were recorded in each of the four anterior regions as seen in Figure 1. In the anatomical body frame, these four regions are the $(x>0, y>0, z>0)$, $(x>0, y<0, z>0),(x<0, y<0, z>0)$ and $(x<0$, $y>0, z>0$ ) quadrants. Subjects were instructed to move their wrist along a given direction pointing to a certain point in space somewhere in the middle of the particular region. These motions ranged from a flexed elbow configuration to an extended elbow configuration. Then they were instructed to point in similar directions to record static poses with and without an additional weight held in their hand.

Each trajectory was recorded at least once, some twice, in each region. Two static poses without weights were recorded in each region. One static pose with weights was recorded in each region. The trajectories and static poses were performed sequentially, quadrant by quadrant, clockwise, starting in $(x<0, y>0, z>0)$. Fatigue was prevented by allowing the subjects to rest during the experiments.

\section{Data analysis}

The purpose of recording the trajectories is to verify the hypothesis that every wrist position along a single reaching direction has the same elbow vector direction. Figure 6 shows a plot of the reflective markers for one of the subjects performing a right-arm trajectory in the superior-lateral region on the anterior side of the torso (see Figure 1 for the region definition). In this figure, the " $*$ " points are the two markers on the sternum, the " $x$ " point is the marker approximately at the shoulder center, the "o" point is the marker approximately at the wrist center, the " $\triangleright$ " point is the marker on the lateral side of the elbow and the " $\triangleleft$ " point is the marker on the medial side of the elbow. This figure also only shows a small sampling of the points taken along the trajectory as only every tenth point is plotted.

As seen in Figure 6, the shoulder center and wrist center do not always lay on one constant vector. This is due to several reasons:

1. The shoulder center moves as the arm is extended in different regions due to the shoulder rhythm. This causes the origin point of the reaching direction to not remain fixed. When all points are zeroed using the shoulder center, Figure 6 changes to appear like Figure 7.

2. Even after zeroing the coordinates of each point in relation to the shoulder center, each reaching position does not lead to exactly the same reaching direction. This comes from the difficulty the subjects had in maintaining a constant reaching direction when performing the trajectories. While it is close to a constant vector, there still exists variation in human movement.

3. If the perfect human subject were found and that person followed a constant reaching direction throughout the trajectory, it would still appear to not lay on a constant vector. There would still be experimental error due to discrepancies in the acquisition of the points using the cameras, round-off error when saving the data and errors caused by the markers shifting due to soft tissue effects. These errors will resurface later when producing a quantified description of the shoulder-elbow coupling. 


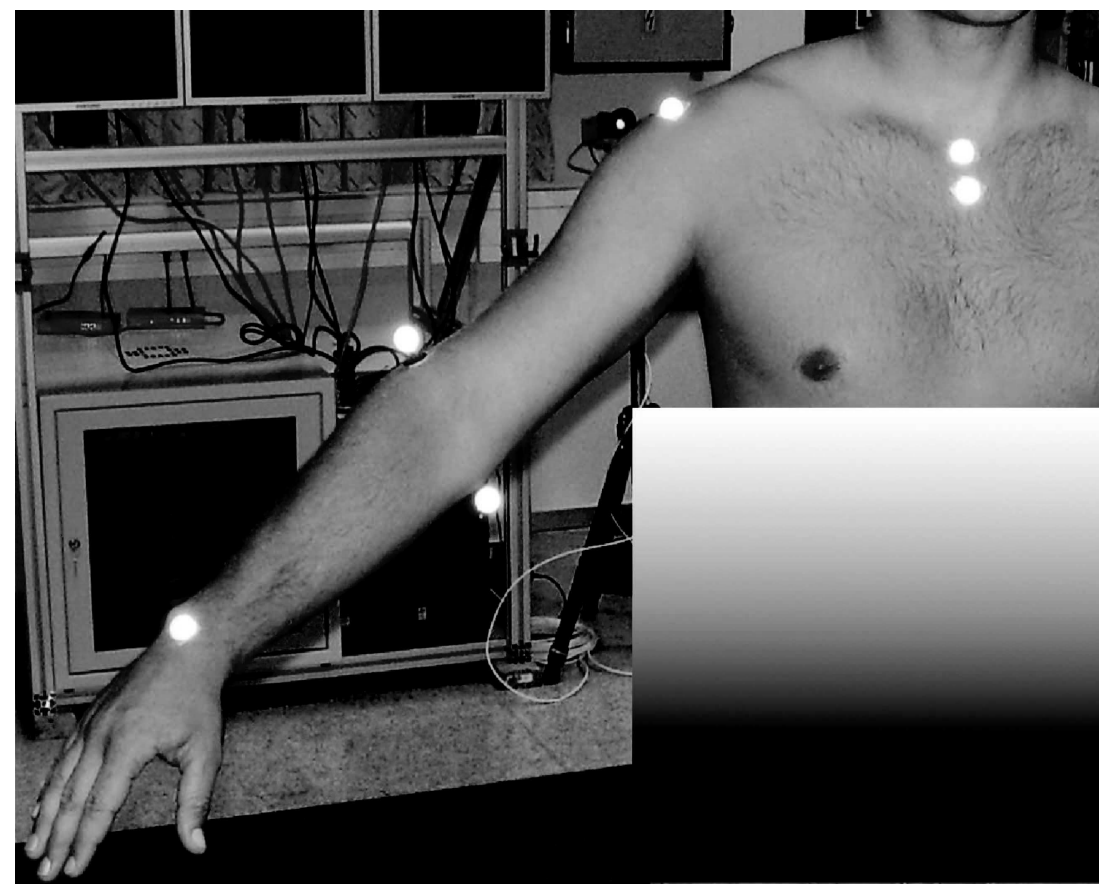

Figure 5 Motion capture marker placements.

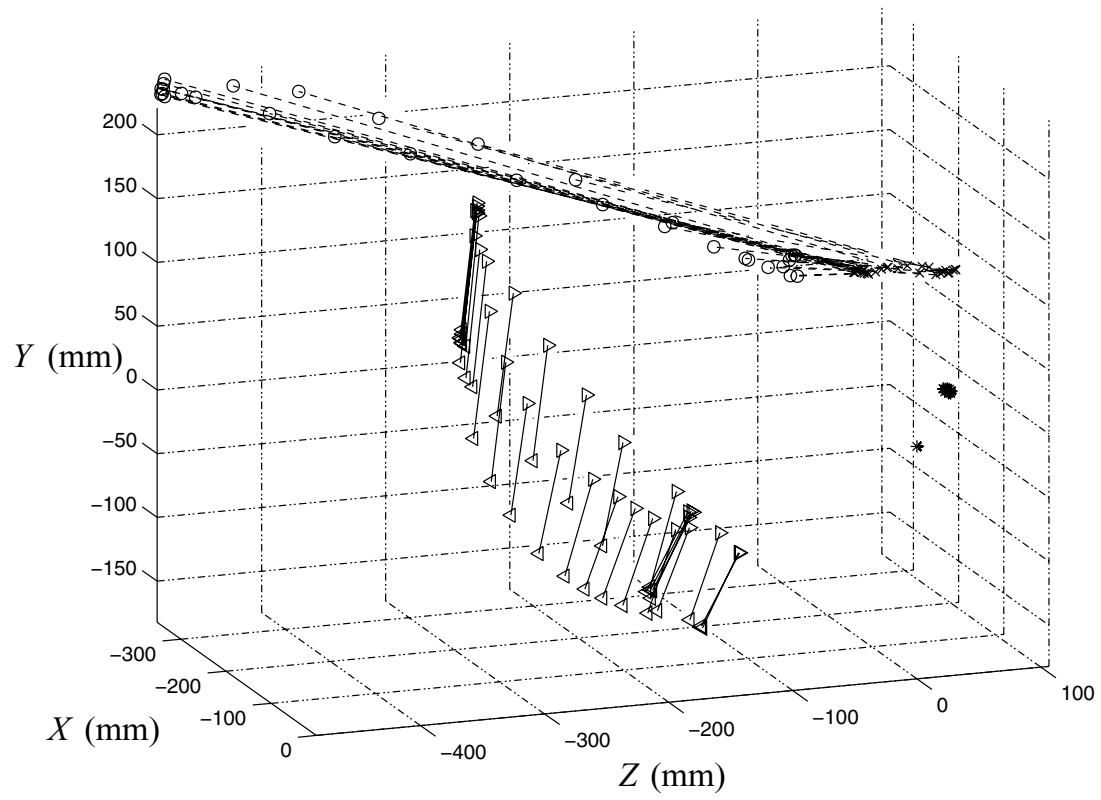

Figure 6 Plot of reflective markers while performing a constant reaching direction trajectory with the coordinates zeroed at the sternum.

Even though an ideal constant reaching direction trajectory was not attained, there were instances for each subject where there existed several data points exhibiting similar reaching directions. It was therefore possible to compare these data points in order to support the hypothesis that each reaching direction had one elbow vector direction. Between 15 and 20 sets of similar points were selected for each subject by hand to be used in the analysis.

The data from each of the eleven subjects performed in the four anterior regions was analyzed to find instances where the reaching directions were similar. The differences between both the reaching directions and their associated elbow vector directions were recorded. This was done by taking the arccosine of the dot product of the two unit vectors in order to find the angular difference. When the reaching directions were within $1^{\circ}$, the mean difference between elbow vector directions was $1.2752^{\circ}$ with a standard deviation of $1.8337^{\circ}$. The large standard deviation can be attributed to the presence of four data points with larger angular differences than the majority of the data pool. This 


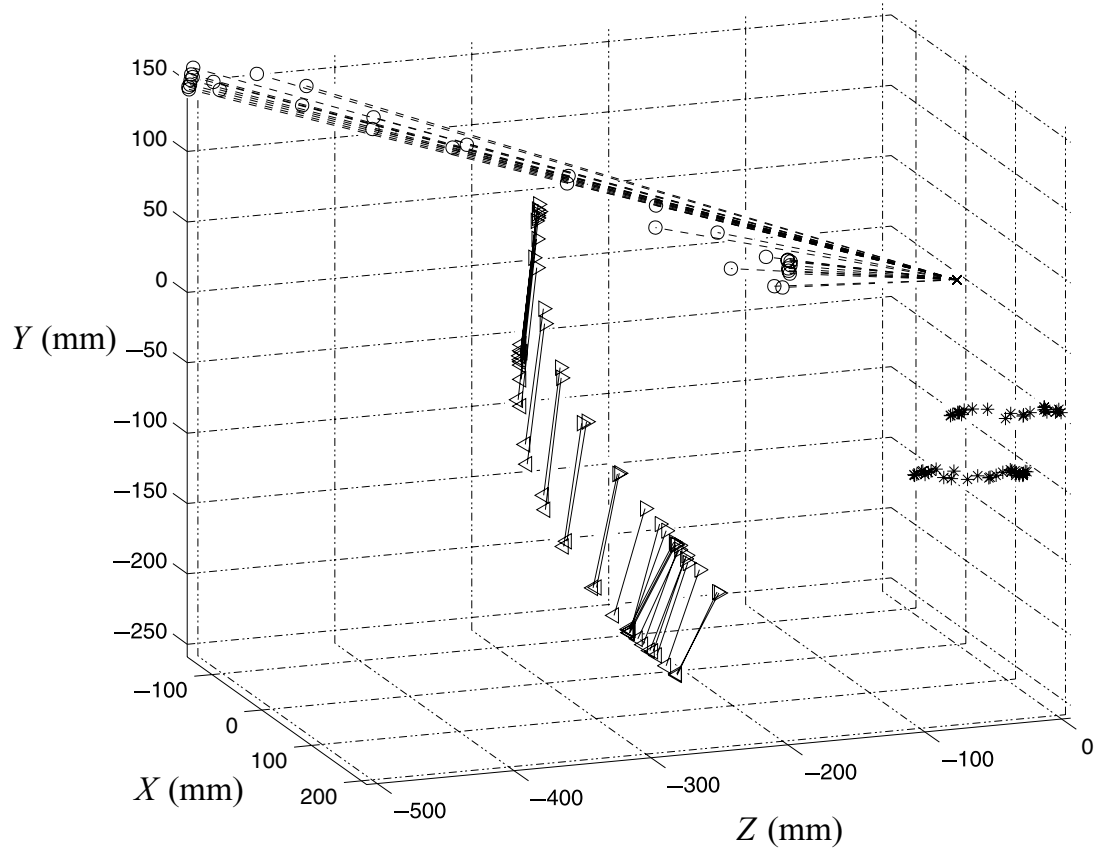

Figure 7 Plot of reflective markers while performing a constant reaching direction trajectory with the coordinates zeroed at the shoulder center.

small mean angular difference between elbow vector directions with near constant reaching directions supports the proposed hypothesis.

\section{Regression analysis}

With the knowledge that the proposed hypotheses are reliable, it is now possible to perform a regression analysis of the data to get surface fits that predict the elbow vector direction when given the reaching direction. In order to get a wide range of reaching directions, each of the eleven subjects performed multiple static poses in the four anterior regions. One pose was also taken from each of the performed trajectories in each region. Thus there were roughly twelve different reaching directions recorded for each of the eleven subjects along with their respective elbow axis directions. There were a few poses that did not have all of the markers register in the cameras and were therefore discarded. Each dataset was zeroed at the shoulder center in order to more easily determine the reaching direction and its respective elbow axis direction.

Finding a good fit that relates scattered data points can often be more of an art than a science. Regression analysis uses a predetermined basis and solves for the coefficients of the desired equation that results in the best approximation of the data. A large set of functions may result in a surface which passes through each data point but is complicated and does not capture the tendencies of the data, while a smaller set may yield a smoother surface which simply approximates the original data. The basis chosen depends on the functions which are appropriate for the data set, whether trigonometric, polynomial, rational, exponential, logarithmic, etc.

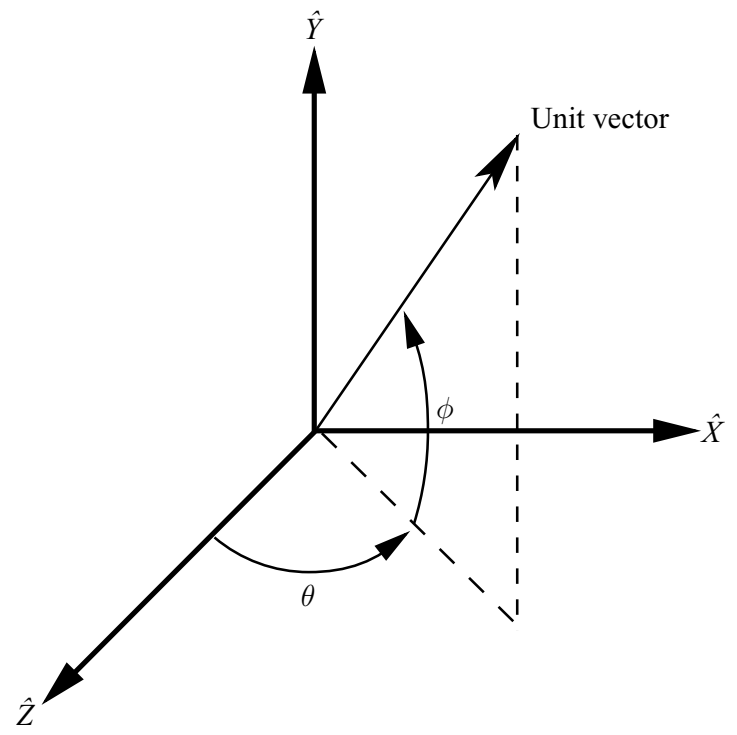

Figure 8 Definition of spherical coordinates.

The data obtained from the eleven test subjects was further processed so that the regression analysis process went smoother. Each unit vector recorded was reduced to two angles, $\phi$ and $\theta$, which represent their spherical coordinates. These angles are defined in Figure 8. Both the reaching direction and elbow vector direction can be decomposed into sets of $\phi$ and $\theta$. It is then possible to relate the two angles of the elbow vector $\left(\phi_{\text {elbow }}\right.$ and $\left.\theta_{\text {elbow }}\right)$ to the two angles of the reaching direction $\left(\phi_{\text {reach }}\right.$ and $\left.\theta_{\text {reach }}\right)$.

Several bases were examined and it was concluded that trigonometric functions best described the relationships between angles. For the surface relating $\phi_{\text {elbow }}$ to $\phi_{\text {reach }}$ and 
Table $1 C_{i j}$ for $\phi_{\text {elbow }}=f\left(\phi_{\text {reach }}, \theta_{\text {reach }}\right)$

\begin{tabular}{lccccc}
\hline & $j=0$ & $j=1$ & $j=2$ & $j=3$ & $j=4$ \\
\hline$i=0$ & 0.124268 & 0.428452 & 0.248536 & -0.475872 & -0.383274 \\
$i=1$ & -0.414052 & 0.0589028 & -1.53609 & 1.22144 & 0.60982 \\
$i=2$ & 0.248536 & -0.752145 & 0.15545 & 2.05931 & -0.762283 \\
$i=3$ & 0.902187 & -0.102347 & 4.56445 & -5.44144 & 0.320791 \\
$i=4$ & 0.48438 & -0.61256 & 0.284034 & -6.18632 & 5.70167 \\
\hline
\end{tabular}

Table $2 D_{i j}$ for $\boldsymbol{\theta}_{\text {elbow }}=\mathbf{g}\left(\phi_{\text {reach }} ; \boldsymbol{\theta}_{\text {reach }}\right)$

\begin{tabular}{lcccccc}
\hline & $j=0$ & $j=1$ & $j=2$ & $j=3$ & $j=4$ & $j=5$ \\
\hline$i=0$ & 0.536725 & 1.60817 & 1.07345 & -7.58161 & 1.34204 & 10.3542 \\
$i=1$ & -0.8035 & -3.92414 & -11.9399 & 33.2265 & 14.1892 & -34.5496 \\
$i=2$ & 1.07345 & -9.23718 & 2.39813 & 42.3229 & 2.90074 & -57.4853 \\
$i=3$ & -2.18068 & 4.40658 & 52.3091 & -125.043 & -42.6398 & 116.649 \\
$i=4$ & -8.25347 & 14.5609 & -8.8459 & -41.9361 & -33.7215 & 88.6117 \\
$i=5$ & 1.64162 & -1.03381 & -47.5504 & 153.448 & -13.5587 & -94.852 \\
\hline
\end{tabular}

$\theta_{\text {reach }}$, the basis took the form of:

$$
\begin{aligned}
\phi_{\text {elbow }}= & \sum_{i=0}^{m} \sum_{j=0}^{m} C_{i j}\left(\sin ^{i}\left(\phi_{\text {reach }}\right) \sin ^{j}\left(\theta_{\text {reach }}\right)\right. \\
& \left.+\cos ^{i}\left(\phi_{\text {reach }}\right) \cos ^{j}\left(\theta_{\text {reach }}\right)\right)
\end{aligned}
$$

where $C_{i j}$ are the constants determined from the regression analysis and $m$ determines the order of the equation. The higher the order, the more closely the surface matches the data but also grows in complexity. Several values of $m$ were examined to determine the average error between the surface and the data points. Also noted was the appearance of the surfaces and how they captured the inherent tendencies of the data points. It was determined that $m=4$ yielded the best combination of low mean error and smoothness of the surface. Table 1 displays the values of $C_{i j}$ for $m=4$. Using the values from Table 1, equation (1) was evaluated at each data point set of $\phi_{\text {reach }}$ and $\theta_{\text {reach }}$. The theoretical value of $\phi_{\text {elbow }}$ obtained from equation (1) was then compared to each corresponding value of $\phi_{\text {elbow }}$ from the data. The resulting mean difference between the surface and the data was $9.3798^{\circ}$ with a standard deviation of $6.9184^{\circ}$. The surface generated from the regression analysis for $\phi_{\text {elbow }}=f\left(\phi_{\text {reach }}, \theta_{\text {reach }}\right)$ is shown in Figure 9.

Again, several bases were examined for the second surface fit. At first, it appeared that polynomial functions resulted in a smooth surface that was able to capture the tendencies of the data. However, it did not produce small errors. Upon further inspection, it was concluded that trigonometric functions again best approximated the relationship between the angular data. Similar to equation (1) the surface relating $\theta_{\text {elbow }}$ to $\phi_{\text {reach }}$ and $\theta_{\text {reach }}$ used the

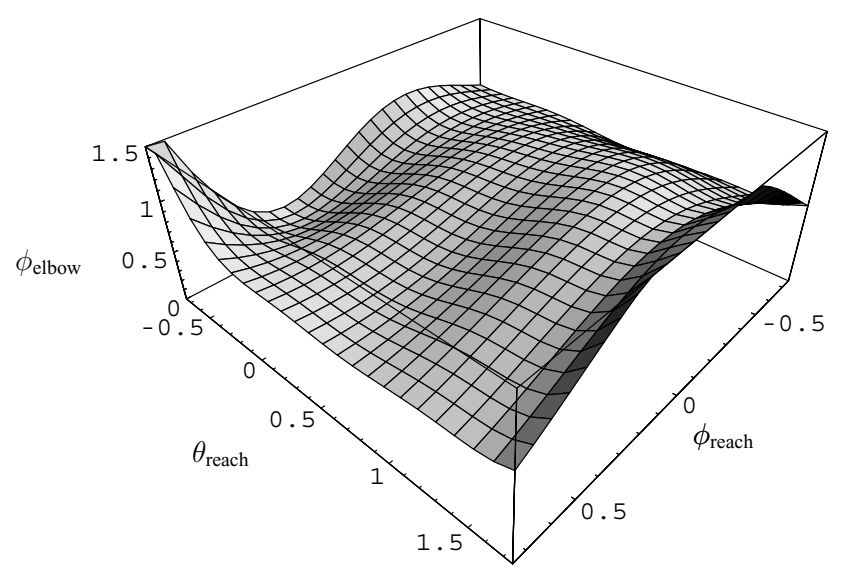

Figure 9 Surface plot of $\phi_{\text {elbow }}=f\left(\phi_{\text {reach }}, \theta_{\text {reach }}\right)$.

basis that took the form of:

$$
\begin{aligned}
\theta_{\text {elbow }}= & \sum_{i=0}^{n} \sum_{j=0}^{n} D_{i j}\left(\sin ^{i}\left(\phi_{\text {reach }}\right) \sin ^{j}\left(\theta_{\text {reach }}\right)\right. \\
& \left.+\cos ^{i}\left(\phi_{\text {reach }}\right) \cos ^{j}\left(\theta_{\text {reach }}\right)\right),
\end{aligned}
$$

where $D_{i j}$ are the constants determined from the regression analysis and $n$ determines the order of the equation. It was determined for this fit that $n=5$ yielded the best combination of low mean error and smoothness of the surface. Table 2 displays the values of $D_{i j}$ for $n=5$. Using the values from Table 2 , equation (2) was evaluated at each data point set of $\phi_{\text {reach }}$ and $\theta_{\text {reach }}$. The theoretical value of $\theta_{\text {elbow }}$ obtained from equation (2) was then compared to each corresponding value of $\theta_{\text {elbow }}$ from the data. The resulting mean difference between the surface and the data was $17.1099^{\circ}$ 


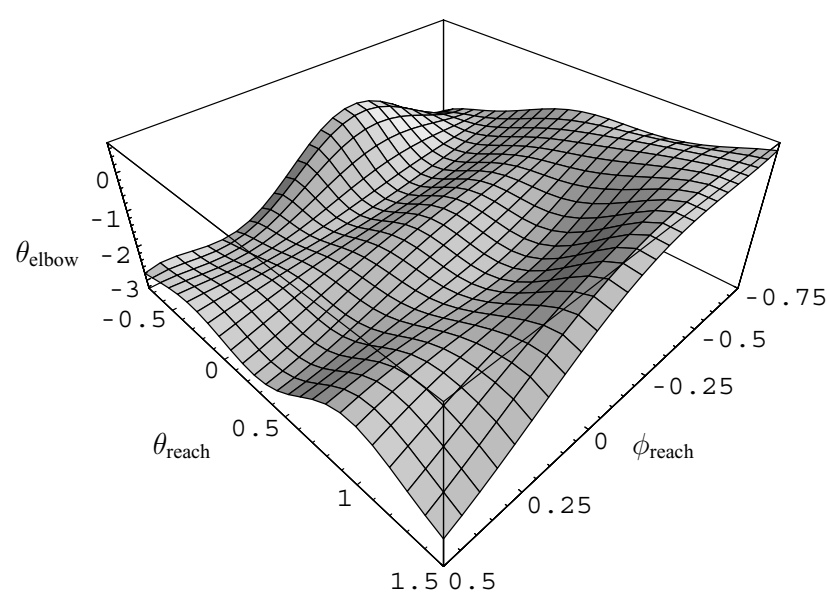

Figure 10 Surface plot of $\theta_{\text {elbow }}=g\left(\phi_{\text {reach }}, \theta_{\text {reach }}\right)$.

with a standard deviation of $13.5334^{\circ}$. The surface generated from the regression analysis for $\theta_{\text {elbow }}=$ $g\left(\phi_{\text {reach }}, \theta_{\text {reach }}\right)$ is shown in Figure 10.

\section{Effect of added weight on the shoulder-elbow coupling}

The question of whether the coupling between the reaching direction and elbow axis direction was developed through some minimization of energy within the shoulder-elbow complex (such as different arm orientations resulting in different allocations of muscular energy) or if it was optimized by the CNS (central nervous system) while developing through adolescence was examined. While this question is not easily answered, an experiment was set up in order to see if adding additional weight to the arm significantly changed the configuration of the arm, therefore suggesting that each time the arm is repositioned the muscular energy within the arm is minimized.

Several additional poses were recorded, this time with each subject holding a $3 \mathrm{~kg}$ mass (weight of $29.42 \mathrm{~N}$ or $6.614 \mathrm{lb}$ ) while performing different reaching poses. This moderate weight was chosen since it does not drastically change the overall weight of the system but changes it enough to notice a difference when positioning the arm. Comparing the data from the poses while holding the weight to the surface fits developed in the previous section results in a mean difference between the $\phi_{\text {elbow }}=f\left(\phi_{\text {reach }}, \theta_{\text {reach }}\right)$ surface and the data of $11.7152^{\circ}$ with a standard deviation of $8.0510^{\circ}$, and a mean difference between the $\theta_{\text {elbow }}=g\left(\phi_{\text {reach }}, \theta_{\text {reach }}\right)$ surface and the data of $22.1003^{\circ}$ with a standard deviation of $14.7891^{\circ}$.

The addition of a small weight slightly changed the orientation of the arm while performing different poses but overall the arm remained in a similar orientation as before. It can be noted that the arm is oriented differently when trying to hold heavier weights such as a stack of books or a tray of food. What would happen to the recorded poses if the small weight was increased by an order of magnitude? Where is the break-off point when the arm starts compensating for the weight and strays from the voluntary arm motions? These questions are both topics for future studies.

\section{Discussion of results}

Both of the surfaces obtained from the regression analysis did qualitatively well at incorporating the tendencies of the data. Quantitatively, it is important to note the existence of experimental error. The sources of this error were mentioned earlier: discrepancies in the acquisition of the points using the cameras, round-off error when saving the data and errors caused by the markers shifting on the skin while performing trajectories.

The error of the recorded 3D position of the markers is declared to normally be less than $\pm 0.5 \mathrm{~mm}$. It depends on the size of the working volume and field of view of the cameras etc., but in this case could not be more than $\pm 1 \mathrm{~mm}$. However, there can exist larger errors due to hidden markers (markers that "vanish" during trajectories or are covered by body parts prohibiting certain cameras from recording their existence), overlapping markers (in several instances there existed multiple recorded markers for the same actual point), detection errors, exchanged trajectories, etc. This was easily recognized because there was a sharp change of the $3 \mathrm{D}$ position. It was concluded that there was roughly a $\pm 1-2 \%$ error in the three-dimensional location of the markers. While only a few millimeters in some cases, it is important to note the effect this error had on calculating $\phi$ and $\theta$. This error in marker positions led to a mean error in the angular data of approximately $\pm 1.5^{\circ}$.

Examining the mean difference and standard deviation of each surface gives an insight on how well the regression analysis did at matching the datasets within error. The surface for $\phi_{\text {elbow }}=f\left(\phi_{\text {reach }}, \theta_{\text {reach }}\right)$ in equation (1) had a mean difference of $9.3798^{\circ}$ with a standard deviation of $6.9184^{\circ}$. While this difference could have been lowered by increasing the order of the system higher than $m=4$, it also got more complex and started to lose the tendencies of the data. The surface for $\theta_{\text {elbow }}=g\left(\phi_{\text {reach }}, \theta_{\text {reach }}\right)$ in equation (2) had a mean difference of $17.1099^{\circ}$ with a standard deviation of $13.5334^{\circ}$. This difference was much higher than that of the previous surface, which was mostly due to the existence of five points that were not near the rest of the data points but still followed the inherent tendencies of that data. Again this difference could have been lowered by increasing the order higher than $n=5$, but the chosen surface fit best approximated the data qualitatively. Looking at these results while considering the experimental error shows that at certain data points the surface was close to exactly matching the values of $\phi_{\text {elbow }}$ and $\theta_{\text {elbow }}$ and at other points exhibited a large difference between the theoretical and actual values.

The mean difference and standard deviation of each surface compared to the poses while holding a weight 
can help understand the nature of the coupling. For the $\phi_{\text {elbow }}=f\left(\phi_{\text {reach }}, \theta_{\text {reach }}\right)$ surface, there was a mean difference of $11.7152^{\circ}$ with a standard deviation of $8.0510^{\circ}$. These numbers are close to the original mean difference of $9.3798^{\circ}$ and original standard deviation of $6.9184^{\circ}$, both off by only a couple of degrees. For the $\theta_{\text {elbow }}=g\left(\phi_{\text {reach }}, \theta_{\text {reach }}\right)$ surface, there was a mean difference of $22.1003^{\circ}$ with a standard deviation of $14.7891^{\circ}$. These numbers are also close to the original mean difference of $17.1099^{\circ}$ and original standard deviation of $13.5334^{\circ}$, with the means off by roughly $5^{\circ}$. When the experimental error is taken into account, these numbers are near identical for $\phi_{\text {elbow }}$ and within a couple of degrees for $\theta_{\text {elbow }}$ suggesting that additional weight does not have a significant impact on the orientation of the arm. Therefore, it was concluded that the coupling between the reaching direction and the elbow axis direction while performing voluntary arm movements even with the addition of moderate weights is primarily developed through the CNS.

\section{CONCLUSION}

This paper has examined the relationship between the motion of the shoulder in pointing the center of the wrist and the resulting orientation of the arm. It was shown that all wrist center positions along one reaching direction effectively have the same elbow axis direction. Surface fits relating the angles defining the elbow axis direction to those of the reaching direction were presented and discussed. The results from this paper are useful in the design and control of shoulder-arm-elbow complex models. These results state that given a reaching direction defined by $\left(\phi_{\text {reach }}, \theta_{\text {reach }}\right)$, the elbow axis vector defined by $\left(\phi_{\text {elbow }}, \theta_{\text {elbow }}\right)$ can be calculated using the surface fit equations $\phi_{\text {elbow }}=f\left(\phi_{\text {reach }}, \theta_{\text {reach }}\right)$ and $\theta_{\text {elbow }}=g\left(\phi_{\text {reach }}, \theta_{\text {reach }}\right)$ displayed in equations (1) and (2), respectively. A future paper will implement these relationships in the control of a humanoid shoulder-arm-elbow complex.

\section{REFERENCES}

Bosga J, Meulenbroek RGJ, Swinnen SP. 2003. Stability of inter-joint coordination during circle drawing: Effects of shoulder-joint articular properties. Human Movement Sci, 22:297-320.

Cruse H, Brüwer M. 1987. The human arm as a redundant manipulator: The control of path and joint angles, Biol Cybernet, 57:137-144.

de Groot JH, Brand R. 2001. A three-dimensional regression model of the shoulder rhythm, Clin Biomech, 16:735-743.

Debicki DB, Gribble PL. 2004. Inter-joint coupling strategy during adaptation to novel viscous loads in human arm movement, $\mathcal{7}$ Neurophysiol, 92:754-765.

Flash T, Hogan N. 1985. The coordination of arm movements: An experimentally confirmed mathematical model, 7 Neurosci, 5(7):1688-1703

Gielen CCAM, Vrijenhoek EJ, Flash T, Neggers SFW. 1997. Arm position constraints during pointing and reaching in 3-D space, 7 Neurophysiol, 78:660-673.

Kieffer J, Lenarcic J. 1994. On the exploitation of mechanical advantage near robot singularities, Informatica, 18:315-323.

McCabe SJ, Goldman S. 2000. The Hand, Wrist, and Arm Sourcebook, Los Angeles: Lowell House.

Novotny JE, Nichols CE, Beynnon BD. 1998. Normal kinematics of the unconstrained glenohumeral joint under coupled moment loads, 7 Shoulder Elbow Surg, 7(6):629-639.

Oatis CA. 2004. Kinesiology: The Mechanics and Pathomechanics of Human Movement, Philadelphia: Lippincott, Williams and Wilkins.

Potkonjak V, Popović M, Lazarević M, Sinanović J. 1998. Redundancy problem in writing: From human to anthropomorphic robot arm, IEEE Trans Systems, Man, Cybernet-Part B: Cybernetics, 28(6):790-805.

De Sapio V, Warren J, Khatib O. 2006. Predicting reaching postures using a kinematically constrained shoulder model, Advances in Robot Kinematics, Ljubljana, Slovenia, June, pp. 209-218.

Zaal F T J M, Daigle K, Gottlieb G L, Thelen E. 1999. An unlearned principle for controlling natural movements, The American Physiological Society, pp. 255-259.

Zatsiorsky V M. 1998. Kinematics of Human Motion, Champaign Human Kinetics. 

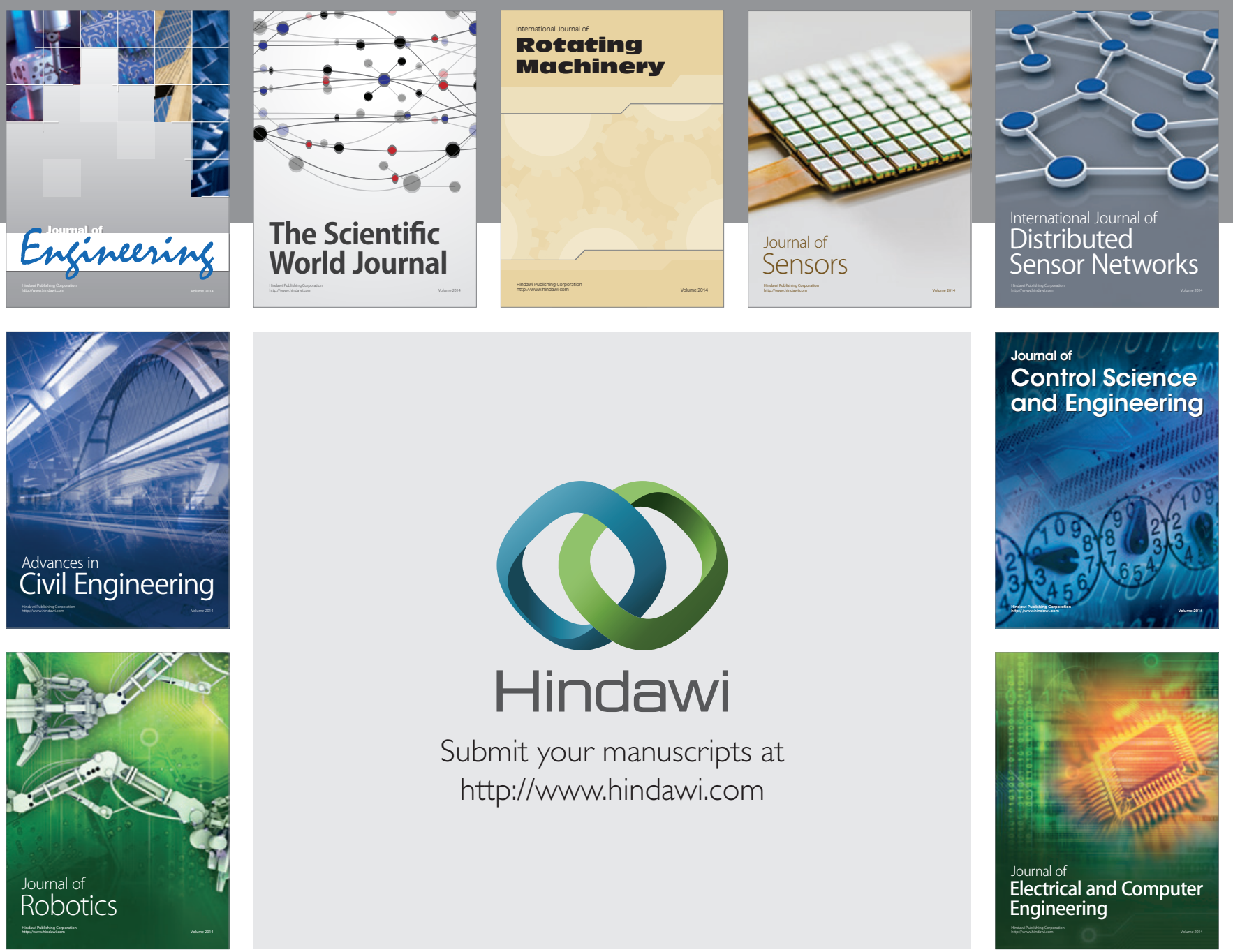

Submit your manuscripts at

http://www.hindawi.com
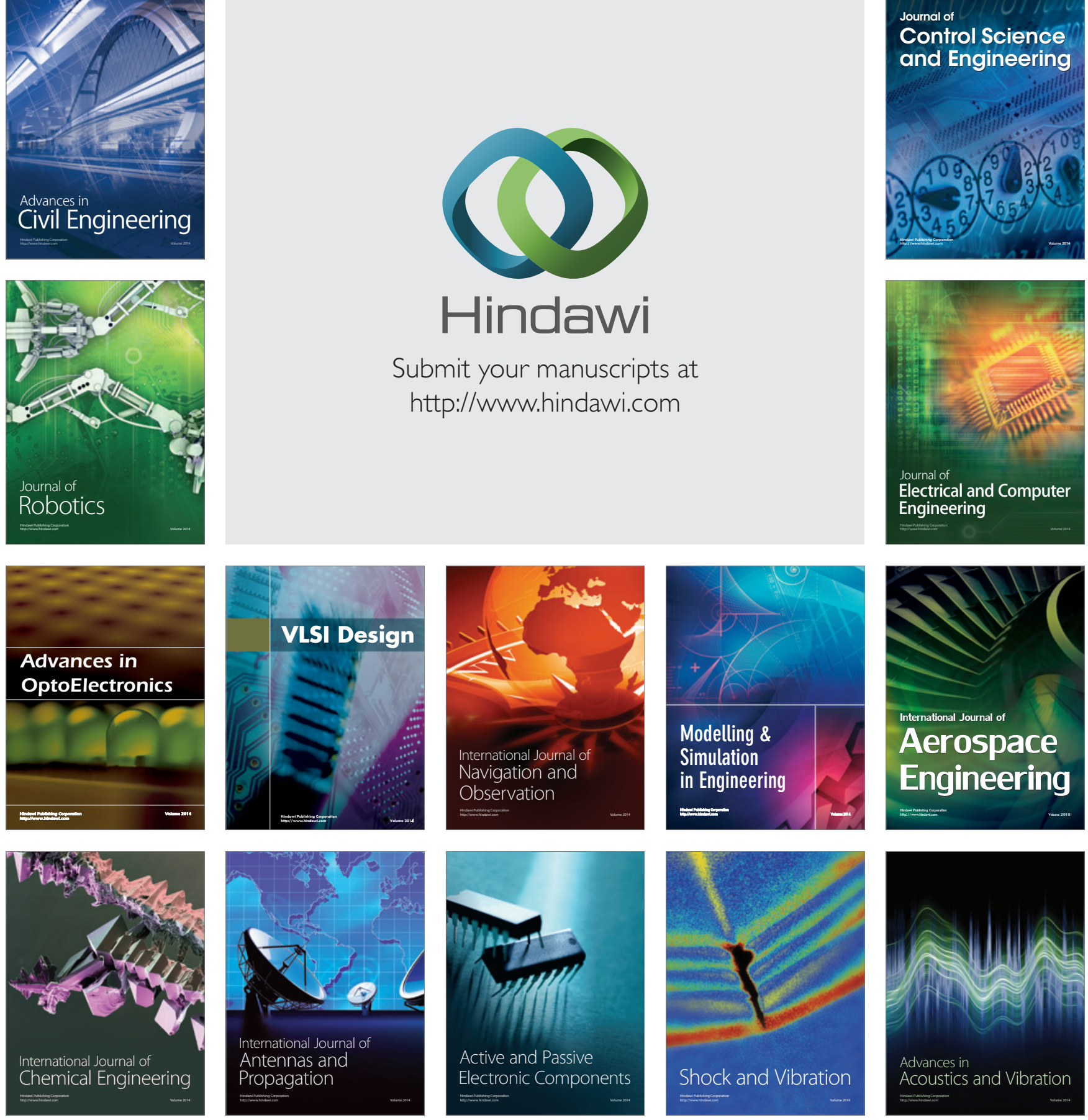